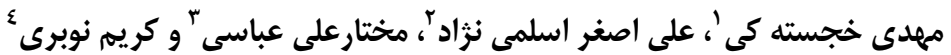

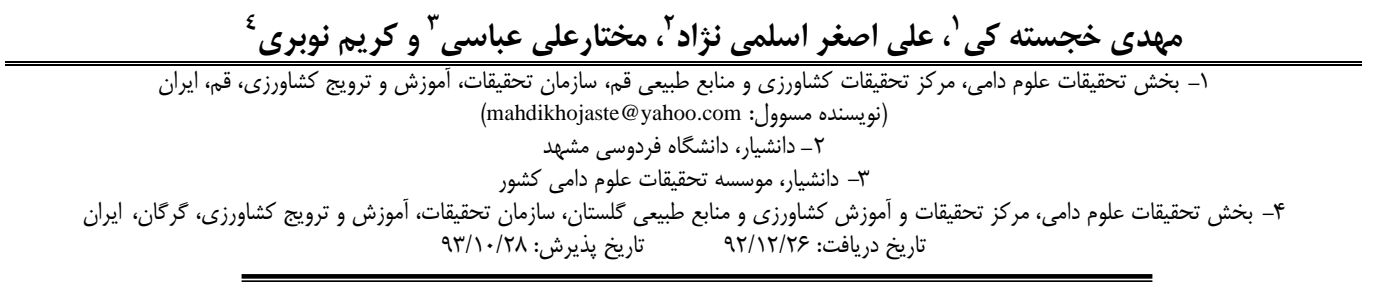

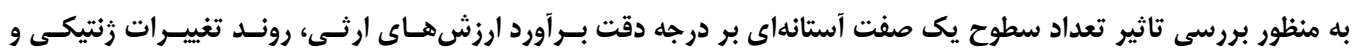

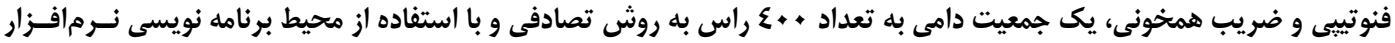

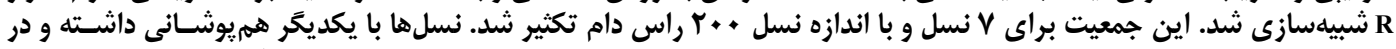

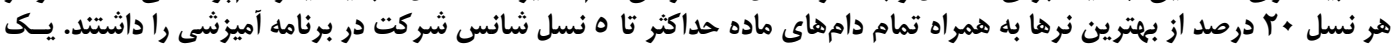

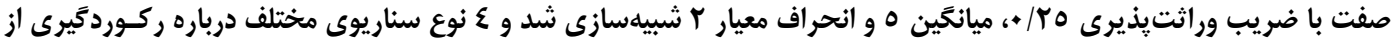

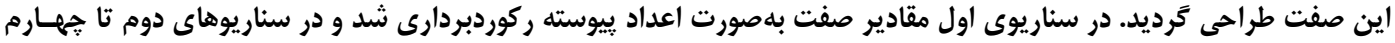

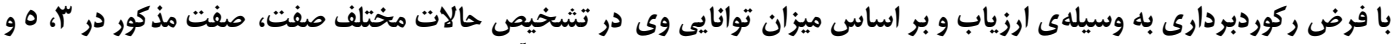

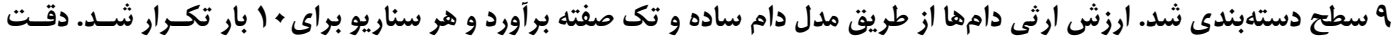

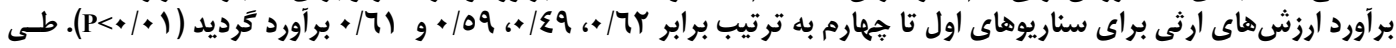

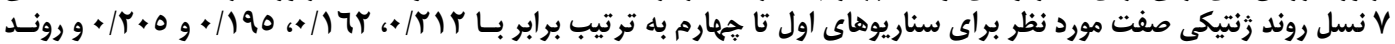

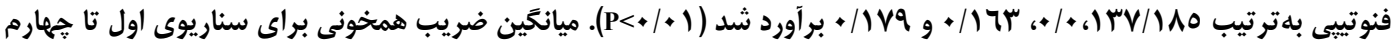

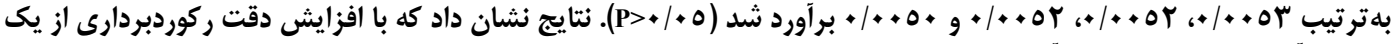

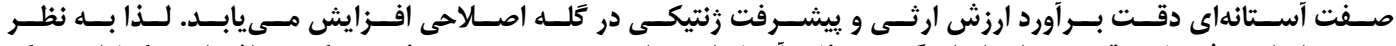

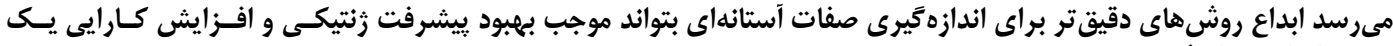

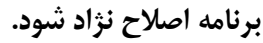

وازههاى كليدى: صفت آستانهاى، ركورد بردارى، ييشرفت زنتيكى، ضريب همخونى

اطلاعات كمى ييوسته مربوط به توزيع زيرين اين گونه صفات

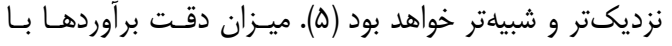

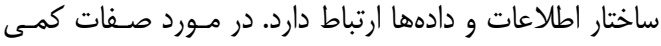

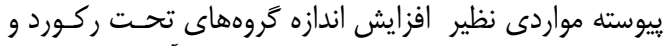

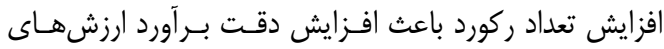

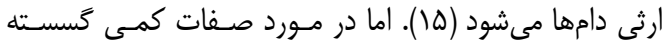

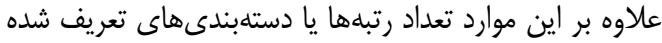

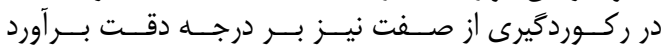

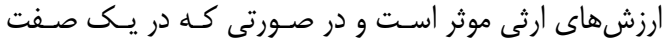

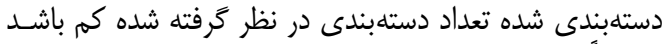

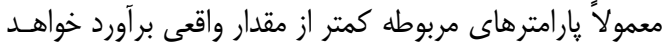

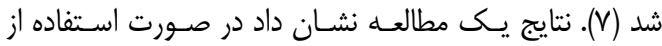

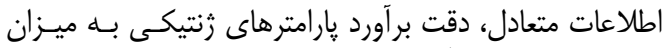

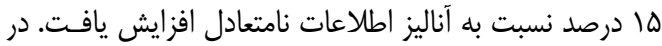

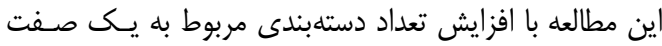

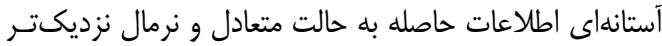

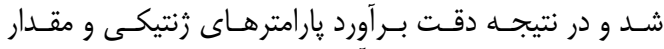

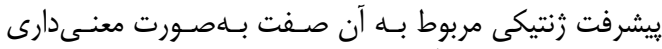

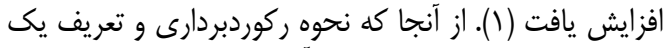

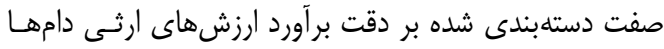

مقدمه

در اصلاح نزاد برخى از صفات مهرم اقتصادى راد در گـروه

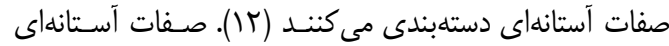

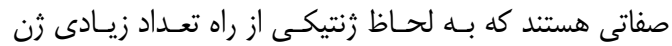

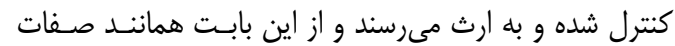

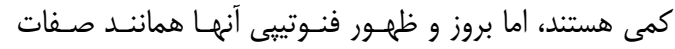

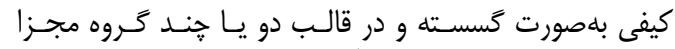

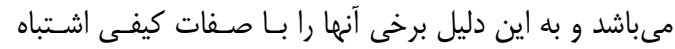

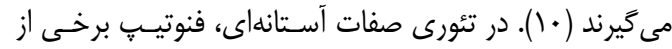

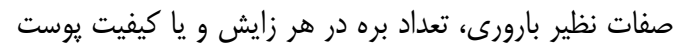

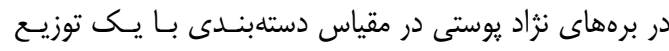

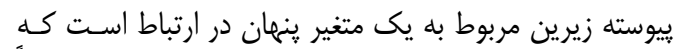

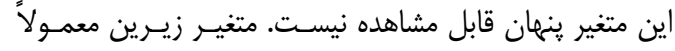

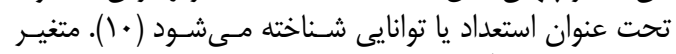

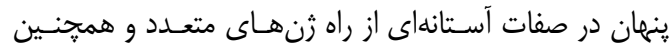

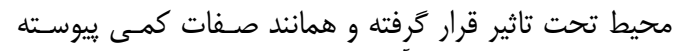

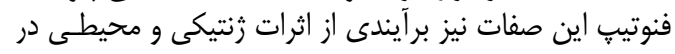

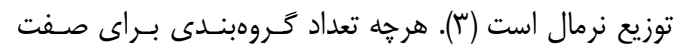

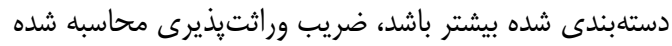

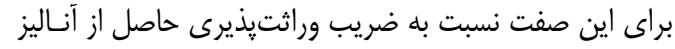




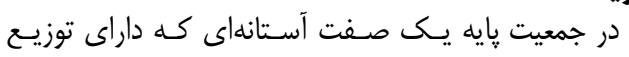

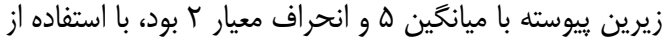

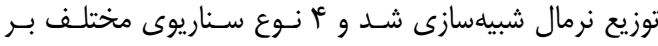

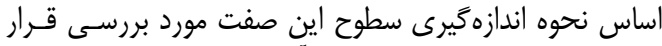

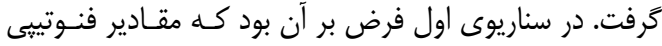

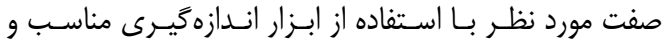

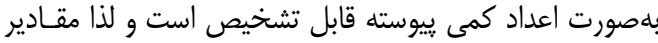

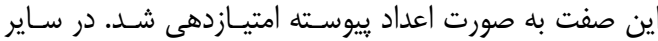

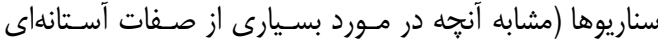

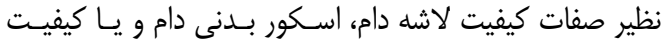

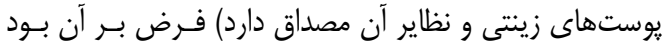

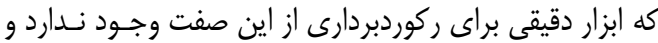

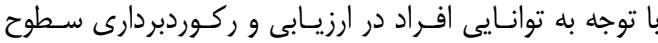

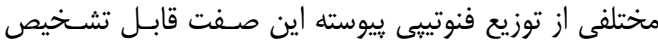

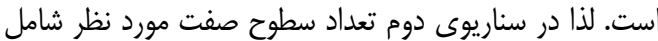

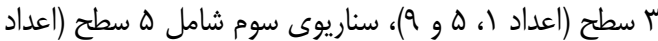

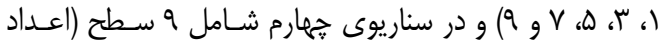

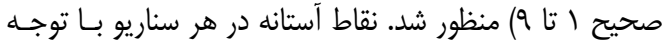

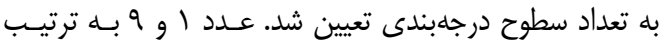

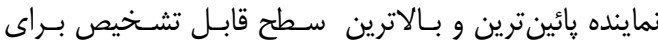

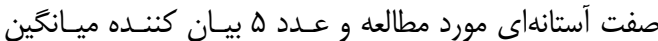

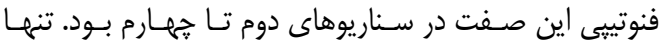

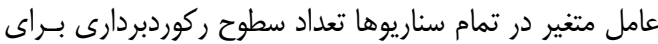
صفت آستانهاى بود.

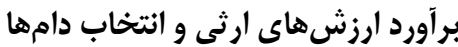

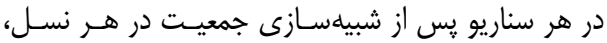

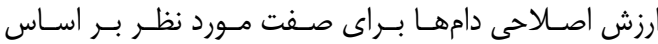

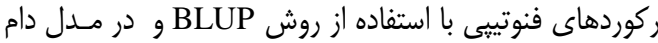

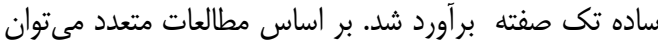

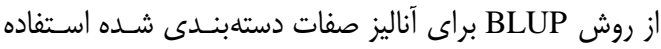

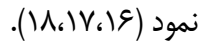

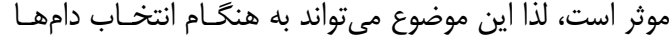

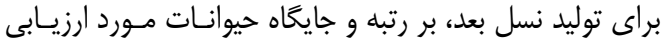

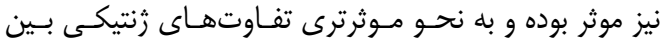

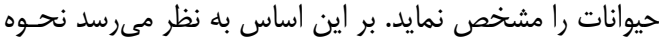

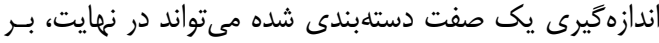

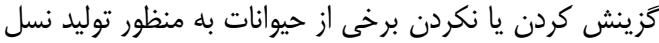

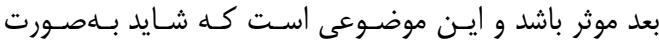

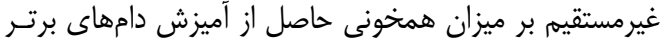
در نسل هاى آتى موثر باشد.

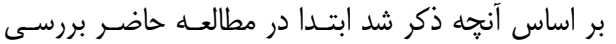

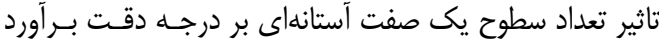

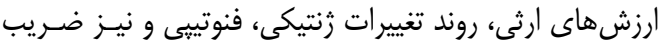

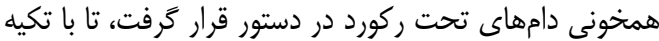

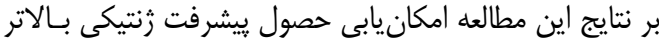

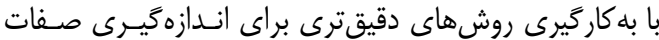

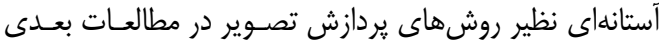

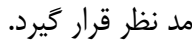

\section{مواد و روشها نها شبيلسازى جمعيت}

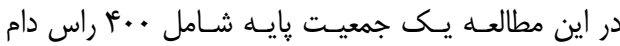

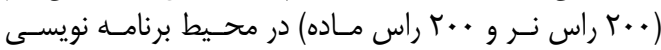

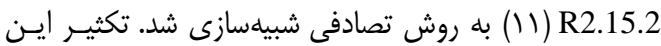

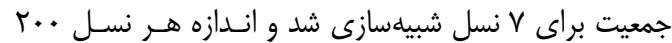

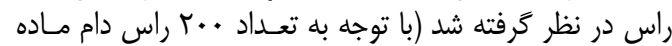

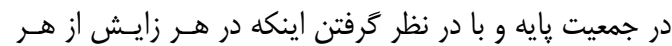

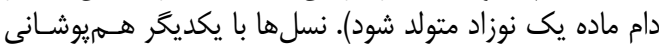

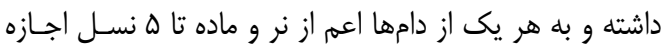

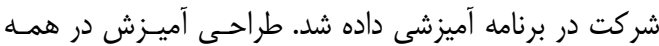

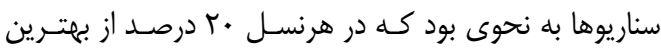

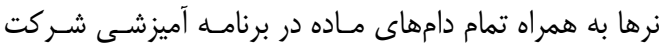

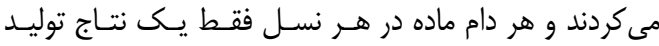

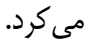

جدول 1- اطلاعات توصيفى مربوط به سناريوهاى مختلف مورد بررسى Table 1. Descriptive information about the various studied scenarios

\begin{tabular}{|c|c|c|c|c|}
\hline سناريوى جهارم & سناريوى سوم & سناريوى دوم & سناريوى اول & \\
\hline$\cdot / T \Delta$ &.$/ T \Delta$ &.$/ T Q$ &.$/ T Q$ & ضريب وراثتيذيرى \\
\hline$\Delta$ & $\Delta$ & $\Delta$ & $\Delta$ & ميانكين فنوتييى \\
\hline r & $r$ & r & r & انحراف معيار فنوتيبى \\
\hline 9 & $\Delta$ & r & ييوسته & تعداد سطوح قابل تشخيص \\
\hline f.. & $f \cdots$ & $f \cdots$ & f.. & تعداد دامها در جمعيت مبنا \\
\hline$r+$. & $r \cdot$. & $r+$. & $r+$. & اندازه هرنسل \\
\hline$\checkmark$ & $\checkmark$ & $\checkmark$ & $\checkmark$ & تعداد نسل \\
\hline 1. & 1. & 1. & 1. & تعداد تكرار هر سناريو \\
\hline
\end{tabular}

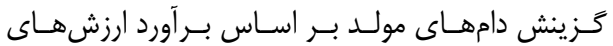

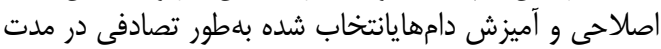

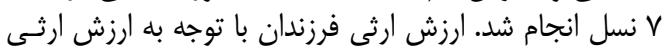

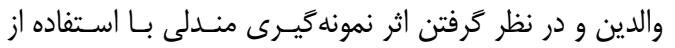
$g_{o}=1 / 2 g_{s}+1 / 2 g_{d}+g_{m}$
مدل آمارى مورد استفاده بـراى بــرآورد ارزش زهــــ ارثـى بــــ

$\mathrm{Y}=\mathrm{m}+\mathrm{a}_{\mathrm{i}}+\mathrm{e}_{\mathrm{ij}}$

شرح زير بود: مداري

در رابطه فوق y عبارت است از هر يك از مشـاهدات،

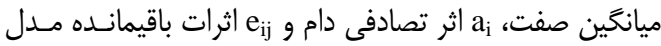
مىباشند. 


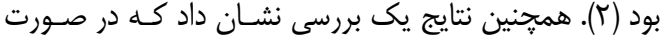

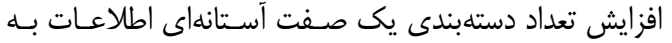

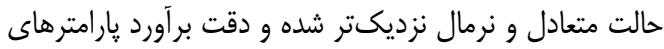

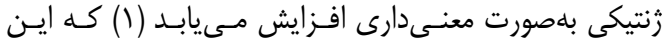

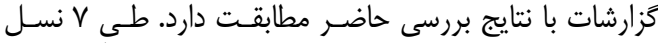

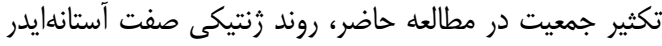

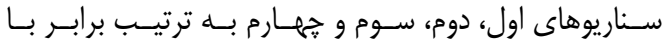
(P</

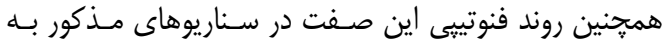

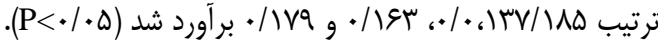

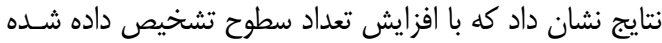

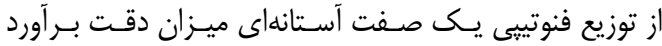

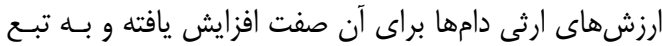

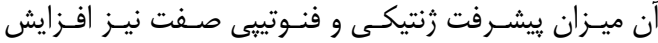

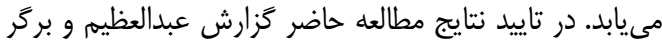

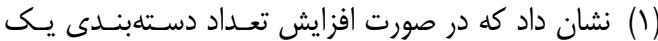

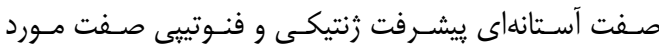

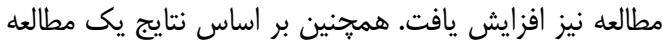

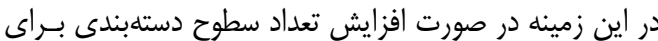

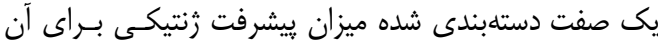

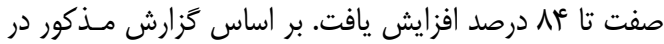

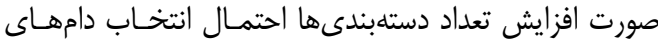

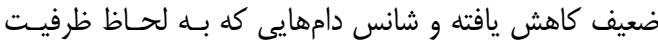

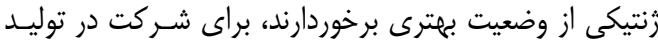

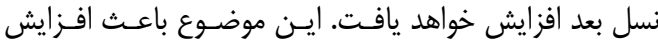

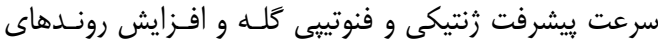

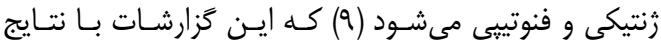
بررسى حاضر مطابقت دارد.

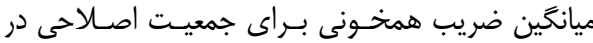

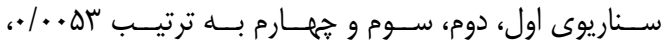

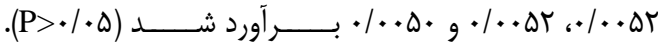

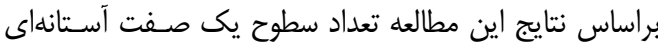
تاثيرى بر ميزان همخونى ايجاد اين مدال نده در كله نداد نداشت

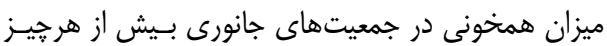

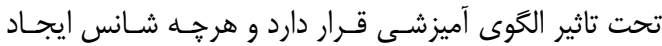

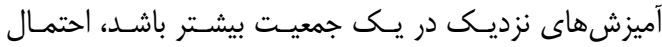

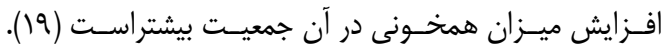

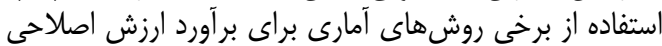

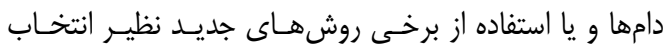

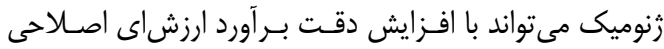

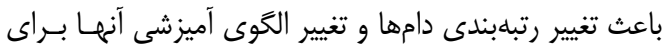

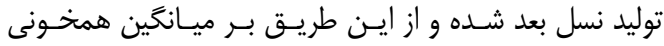

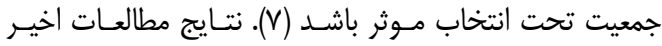

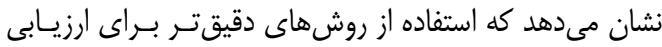

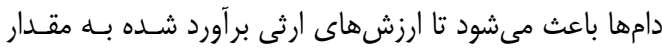

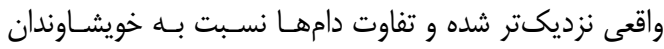

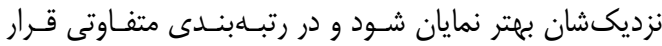

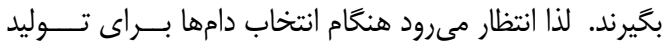

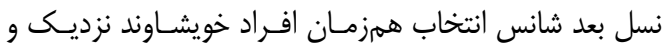

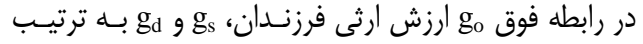

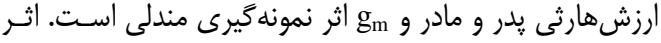
نمونه

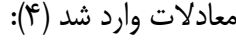

$\sigma^{2} \mathrm{~g}_{\mathrm{m}}=1 / 2\left[1-1 / 2\left(F_{s}+F\right)\right] * \sigma^{2} \mathrm{~g}_{0}$

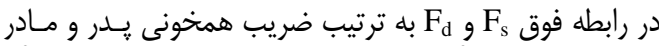

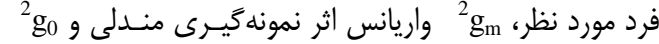
واريانس ارزش ارثى فرزندان مى فياشد.

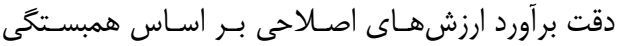

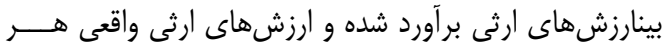

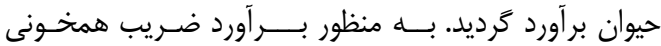

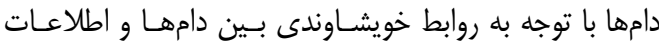

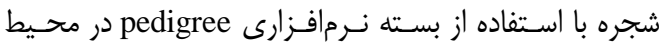

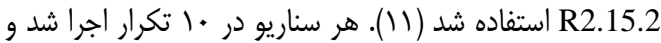
خروجى آن بـا اسـتفاده از روش

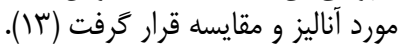

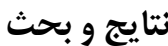

نتايج مربوط به مقايسه ميانگين يارامترهاى برآورد شده در

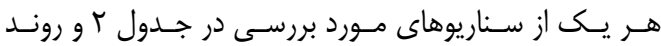

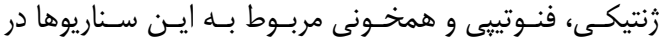
شكلهاى ا تا س ارائه شده است.

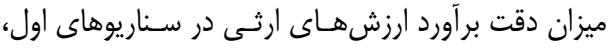

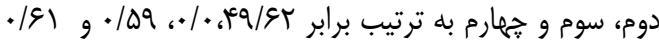

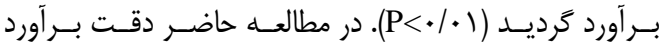

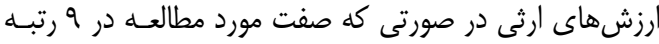

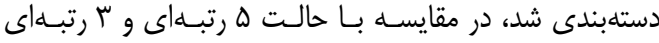

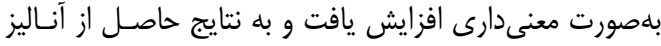

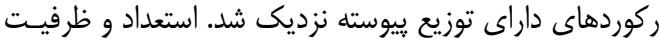

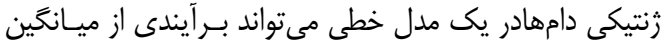

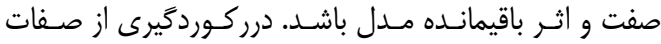

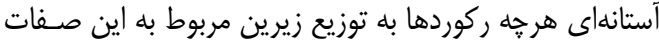

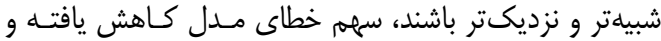

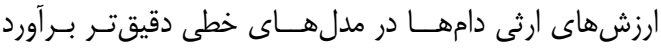

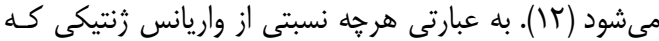

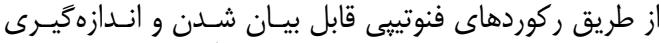

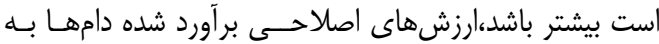

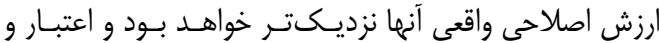

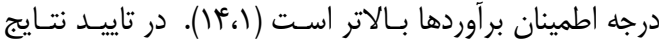

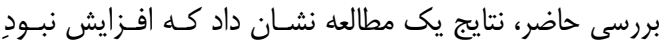

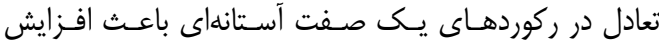

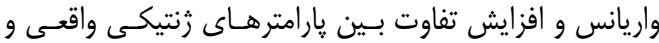

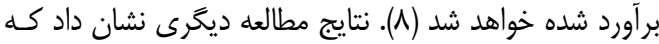

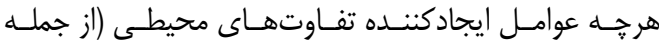

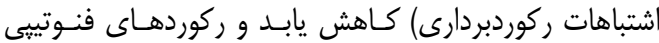

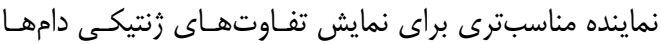

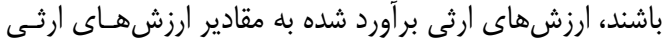

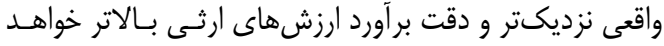




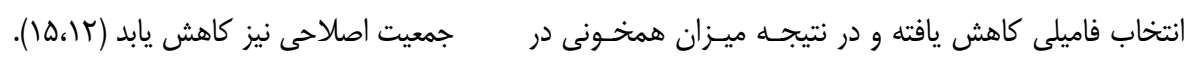

Table 2. The mean and standard error of studied parameters of threshold trait

\begin{tabular}{|c|c|c|c|c|c|}
\hline P-value & سناريوى خهارم & سناريوى سوم & سناريوى دوم & سناريوى اول & متغير مورد بررسى \\
\hline.$/$. & $\Delta / F^{c} \pm \pm \cdot / \cdot 1 Q^{b}$ & $Q / \mathcal{F} T \pm \cdot / \cdot 1 \mathcal{G}^{\mathrm{b}}$ & $\Delta / r I \pm \cdot / \cdot r I^{c}$ & $\Delta / \Delta \Psi_{ \pm} \cdot / \cdot 1 \omega^{\mathrm{a}}$ & ميانخين فنوتييى \\
\hline. & $\cdot / 4 \mu_{ \pm} \cdot / \cdot \cdot \Lambda^{b}$ & $\cdot / \mathrm{Ar}_{ \pm} \cdot / \cdot \cdot \Lambda^{\mathrm{b}}$ & $\cdot / q_{ \pm} \cdot / \cdot \cdot \wedge^{c}$ & $\cdot \mid \Delta \Delta \pm \cdot / \cdot \cdot \wedge^{\mathrm{a}}$ & ميانگين ارزش ارثى واقعى \\
\hline. & $\cdot / q \mathrm{~V} \pm \cdot / \cdot \cdot q^{\mathrm{b}}$ & $\cdot \mid \phi^{c} \pm \cdot / \cdot \Delta^{c}$ & $\cdot \mid \Delta r_{ \pm} \cdot / \cdot \Delta^{d}$ & $\cdot \mid \varepsilon_{ \pm}+1 \cdot \cdot q^{a}$ & ميانگين ارزش ارثى برآورد شده \\
\hline .1. & $\cdot|q| \pm \cdot / \cdots \cdot r^{b}$ & $\cdot / \Delta q \pm \cdot / \cdots+r^{c}$ & $\cdot / q_{ \pm} \cdot / \ldots r^{d}$ & $\cdot \mid q r \pm \cdot 1 \cdot \cdots r^{\mathrm{a}}$ & درجه دقت برآورد ارزش ارثى \\
\hline - IATD & $\cdot / \cdot \cdot \cdot \pm \cdot / \cdots \cdot r^{a}$ & $\cdot / \cdot \Delta r_{ \pm} \cdot / \cdots r^{a}$ & $\cdot / \cdot \Delta r \pm \cdot / \cdots r^{a}$ & $\cdot / \cdot \Delta \pm \cdot / \cdots r^{\mathrm{a}}$ & ميانگين همخونى \\
\hline$\cdot 1+\cdot 1$ & $\cdot / r \cdot \Delta \pm \cdot / \cdots r^{a}$ & $\cdot / 19 \Delta \pm \cdot / \cdots r^{\mathrm{a}}$ & 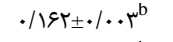 & $\cdot / t \mid r \pm \cdot / \cdot r_{a}$ & روند زنتيكى \\
\hline שז./. & $\cdot / 1 \vee q_{ \pm} \cdot / \cdot r^{a}$ & $. / \triangle q_{ \pm} \pm \cdot / . \kappa^{\mathrm{ab}}$ & $\cdot / \mathrm{HV}_{ \pm} \cdot / \cdot \cdot \varphi^{\mathrm{b}}$ & $\cdot / \wedge \wedge \Delta \pm \cdot / \cdot \cdot r^{\mathrm{a}}$ & روند فنوتيبى \\
\hline.$/ \Lambda \Delta$ & $\cdot / \cdot r \pm * /\left.\cdots \cdot\right|^{a}$ & $\cdot / \cdot r \pm \cdot / \cdot \cdot)^{a}$ & $\cdot 1 \cdot r \pm \cdot /\left.\cdots\right|^{a}$ & $\cdot / * r \pm * / \cdots 1^{a}$ & روند همخونى \\
\hline
\end{tabular}

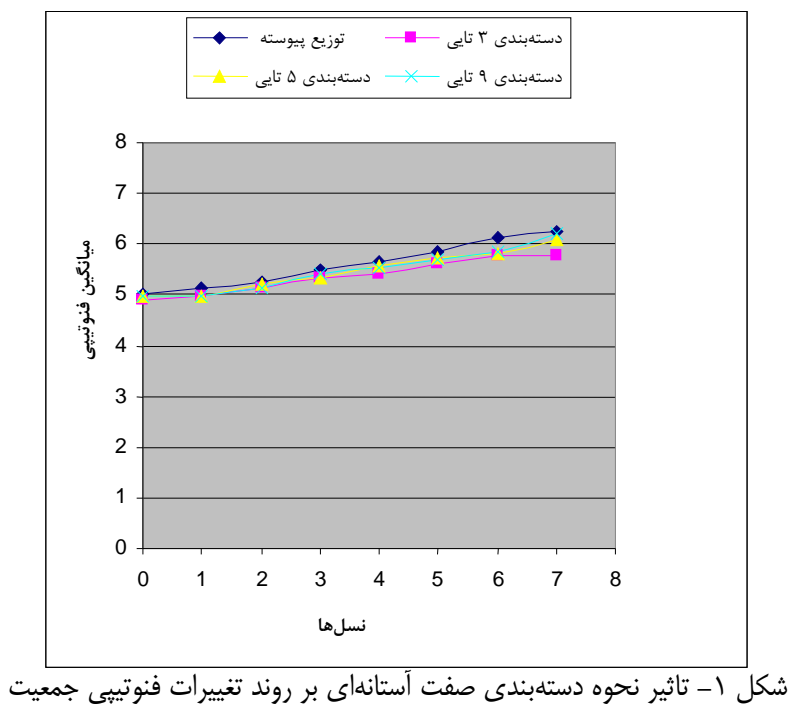

Figure 1. The classification effect of threshold traits on phenotypic trends in population

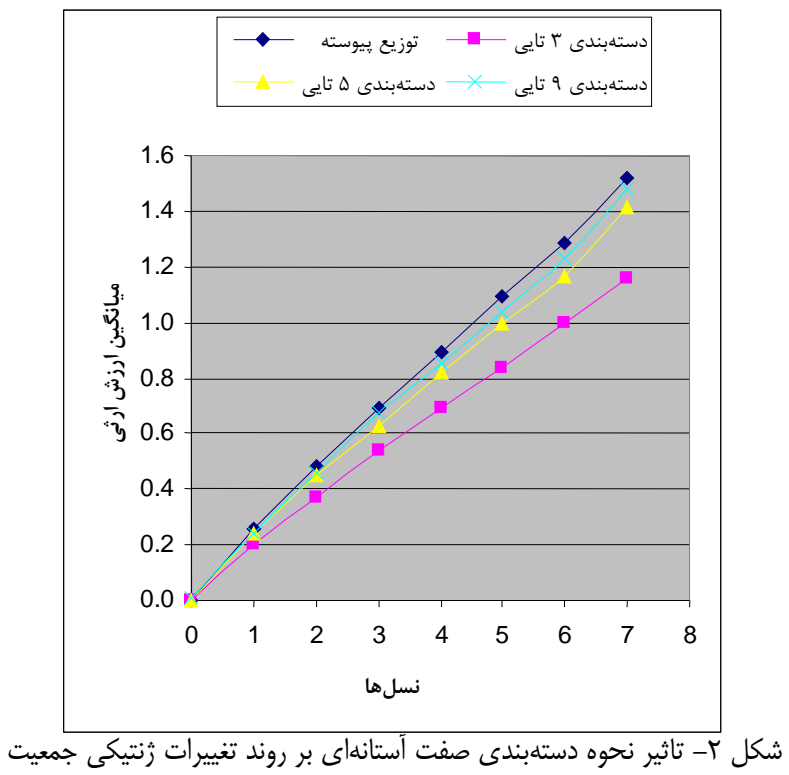

Figure 2. The classification effect of threshold traits on the genetic changes in population 


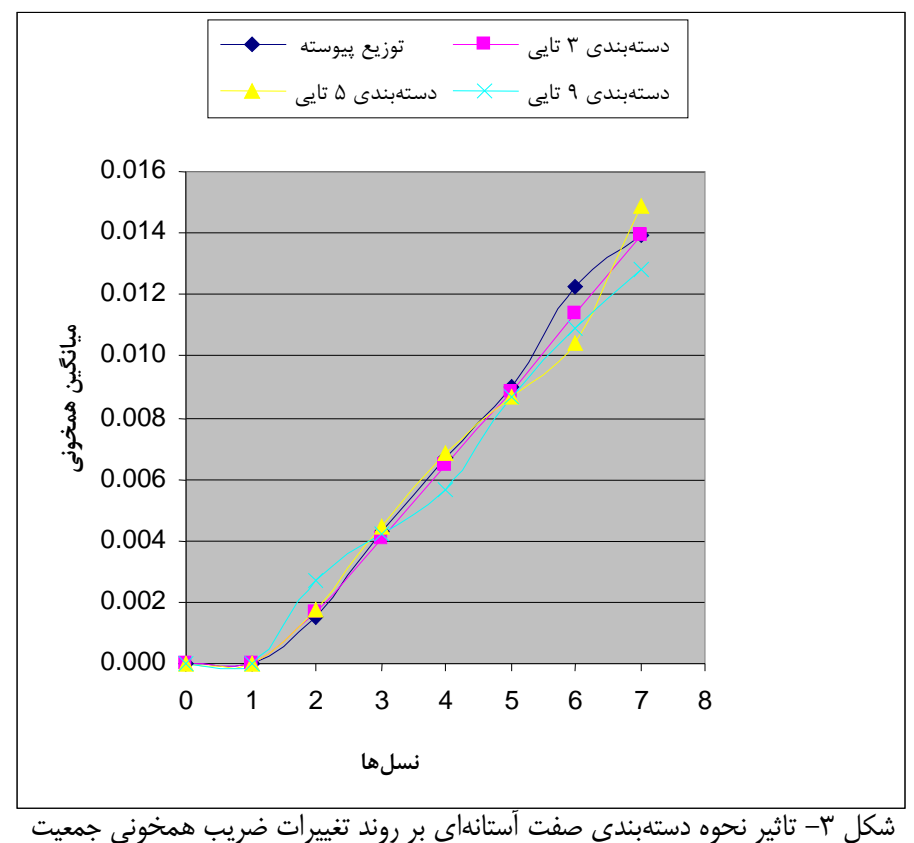

Figure 3. The classification effect of threshold traits on the inbreeding coefficient trends in population

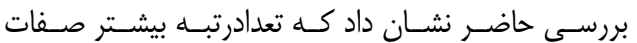

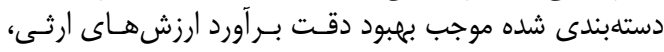

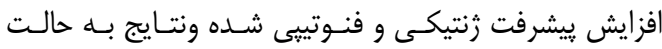

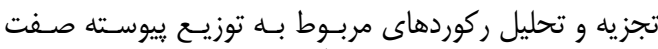

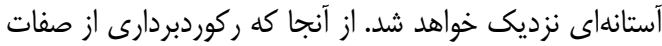

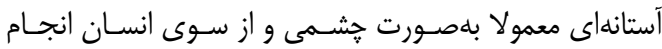

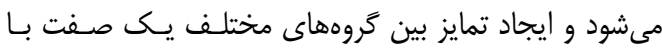

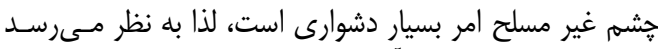

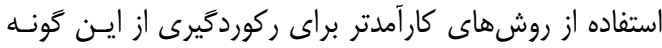

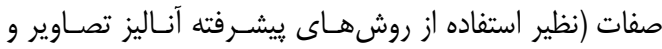

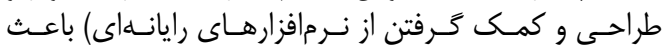

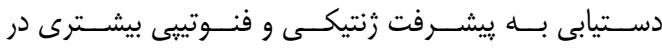
جمعيتهاى اصلاحى خواهد شد.
در بررسى حاضر هرجند در تمام سناريوها تغييرات ميانكَين

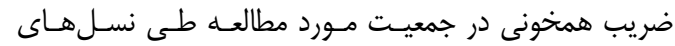
مختلف روند صعودى داشت، اما نتايج نشان داد كه است استفاده از

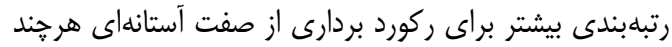

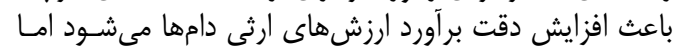

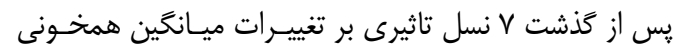

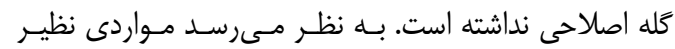

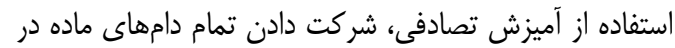

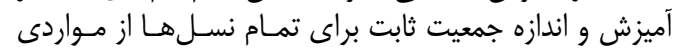

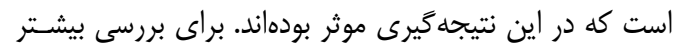

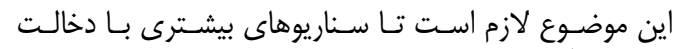

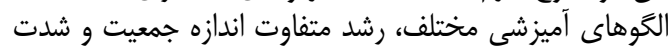
انتخاب متفاوت طراحى و اجرا شود. 
1. Abdelazim A and P J. Berger. 1999. Properties of threshold model predictions. Journal of Anima Science, 77: 582-590

2. Bates, R.O. 1999. Performance records and their use in genetic improvement Purdue Extension. http://www.ces.purdue. edu/extmedia/NSIF/NSIF-5/NSIF-FS5.pdf (12. apr. 2012)

3. Dempster, E.R. and I.M. Lerner. 1950. Heritability of threshold characters Genetics, 35: 212 pp.

4. Falconer, D.S. and T.F.C. Mackay. 1996. Introduction to Quantitative Genetics Longman, Essex, U.K., $4^{\text {th }}$ edition.

5. Gianola, D. 1979. Analysis of discrete variables in animal breeding contexts Journal of Dairy Science, 62: 1471-1478.

6. Gilmour, A.R. 1983. The estimation of genetic parameters for categorical traits Ph.D. Thesis. Massey University New Zealand. $195 \mathrm{pp}$.

7. Hayes, B. and M. Goddard. 2010. Genome-wide association and genomic selection in animal breeding. Genome, 53: 876-883.

8. Hossein-Zadeh, N.Gh. 2007. Effect of the threshold nature of traits on heritability estimates obtained by linear model. Pakistan Journal of Biological Sciences, 10: 145-147.

9. Meuwissen, T.H., B. Engel and J.H. Vanderwerf. 1995. Maximizing selection efficiency for categorical traits. Journal of Animal Science, 73: 1933-1939.

10. Olausson, A. and K. Ronnigen. 1975. Estimation of genetic parameters for there should characters. Acta Agriculture Scandinavia, 25: 1975.

11. R (programming language). 2013. http://cran.r-project.org/web.

12. Rao, Sh. 1997.Genetic analysis of sheep discrete reproductive traits using simulation and field data. Ph.D. Thesis. Virginia Polytechnic Institute and State University, 235 pp.

13. SPSS for Window. Release 16.0. 2007.

14. Stalder, K. 1999. Performance records on relatives. Purdue Extension. http://www.ces.purdue.edu/extmedia/NSIF/NSIF-7/NSIF-FS7. html (12. apr. 2012).

15. Urankar, J., T. Flisar, M. Kovac and S. Malovrh. 2012. Effect of group size on breeding value accuracy in gilts. Acta Agriculture Slovenica, 3: 187-191.

16. Van Vleck, L.D. and K.E. Gregory. 1992. Multiple-trait restricted maximum likelihood for simulated measures of ovulation rate with underlying multivariate normal distributions. Journal of Animal Science, 70: 57-65.

17. Weller, J.I. and M. Ron.1992. Genetic analysis of fertility traits in Israeli Holsteins by linear and threshold models. Journal of Dairy Science, 75: 2541-2549.

18. Wierzbicki, H. 2004. Breeding value evaluation in Polish fur animals: Estimates of direct heritability and portion of litter variation of fur coat and reproduction traits. Czech Journal of Animal Science, 49: 474-482.

19. Woolliams, J.A., R. Pong-Wong and B. Villanueva. 2002. Strategic optimization of short- and longterm gain and inbreeding in MAS and non-MAS schemes. In Proc. $7^{\mathrm{t}}$ World Congress on Genetics Applied to Livestock Production, 33: 155-162. 


\title{
Investigation the Effect of a Threshold Trait Levels on the Accuracy of Breeding Value Estimations and Genetic, Phenotypic and in Breeding Trends in a Breeding Flock
}

\author{
Mahdi Khojastehkey ${ }^{1}$, Ali Asghar Aslaminejad ${ }^{2}$, Mokhtar Ali Abbasi ${ }^{3}$ and Karim Nobari ${ }^{4}$ \\ 1- Animal Science Research Department, Qom Agricultural and Natural Resources Research and Education Center, \\ (AREEO), Qom, Iran (Corresponding author: mahdikhojaste@ yahoo.com) \\ 2- Associate Professor, Ferdowsi University \\ 3- Associate Professor, of Scientific Board of Animal Science Research Institute of Iran \\ 4- Animal Science Research Department, Golestan Agricultural and Natural Resources Research and Education Center, \\ AREEO, Gorgan, Iran \\ Received: March 17, $2014 \quad$ Accepted: January 18, 2015
}

\begin{abstract}
To investigate the effects of categorical trait levels on EBVs accuracies, genetic and phenotypic trends and inbreeding coefficient, a breeding stock contained 400 animals was simulated through stochastic method using $\mathrm{R}$ programming package. The population was simulated for 7 generations while each generation contained 200 animals. The generations were overlapped and in each ones 20 percent of the best males and all of the females had a chance to participate in the mating program up to 5 generations. A categorical trait with heritability of 0.25 , mean of 5 and standard deviation of 2 was simulated, and on the basis of recording approach, 4 scenarios were designed. In the first scenario a threshold trait was measured as continuous records, while for scenarios 2 to 4 , regarding to the appraiser ability a threshold trait were recorded in 3,5 and 9 categories respectively. Breeding values were estimated by a simple, single trait animal model and all scenarios were replicated for 10 times. The accuracy of breeding value estimations for scenarios 1 to 4 were $0.62,0.49,0.59$ and 0.61 respectively $(\mathrm{p}<0.01)$. Through 7 generations, the genetic trends for scenarios 1 to 4 were $0.212,0.162$, 0.195 and 0.205 and the phenotypic trends were $0.185,0.137,0.163$ and 0.179 respectively $(\mathrm{p}<0.01)$.The mean of inbreeding coefficient for scenarios 1 to 4 were estimated 0.0053 , $0.0052,0.0052$ and 0.0050 respectively $(\mathrm{p}>0.05)$. Results showed that, the improvement of recording accuracies for threshold traits lead to increasing in the accuracy of breeding value estimations and the genetic gain. So this is concluded that the development of more accurate measurement tools for threshold traits, could improve the genetic gain and breeding program efficiency.
\end{abstract}

Keywords: Genetic gain, Inbreeding coefficient, Recording, Threshold traits 